\title{
Collective Space-Charge Phenomena in the Source Region
}

\author{
I. Haber ${ }^{a}$, S. Bernal ${ }^{a}$, C. M. Celata ${ }^{b}$, A. Friedman ${ }^{b}$, D. P. Grote ${ }^{b}$,

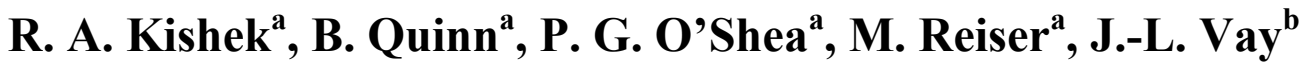 \\ a Institute for Research in Electronics and Applied Physics, University of Maryland, \\ College Park, MD 20742-3511 \\ ${ }^{\mathrm{b}}$ Heavy Ion Fusion - Virtual National Laboratory, Berkeley, CA 97420
}

\begin{abstract}
For many devices space-charge-dominated behavior, including the excitation of space-charge collective modes, can occur in the source region, even when the downstream characteristics are not space-charge-dominated. Furthermore, these modes can remain undamped for many focusing periods. Traditional studies of the source region in particle beam systems have emphasized the behavior of averaged beam characteristics, such as total current, rms beam size, or emittance, rather than the details of the full beam distribution function that are necessary to predict the excitation of collective modes.

A primary tool for understanding the detailed evolution of a space-chargedominated beam in the source region has been the use of simulation in concert with detailed experimental measurement. However, "first-principle" simulations beginning from the emitter surface have often displayed substantial differences from what is measured. This is believed to result from sensitivities in the beam dynamics to small changes in the mechanical characteristics of the gun structure, as well as to similar sensitivities in the numerical methods.

Simulations of the beam in the source region using the particle-in-cell WARP code and comparisons to experimental measurements at the University of Maryland are presented to illustrate the complexity in beam characteristics that can occur in the source region. In addition, direct measurement of the beam characteristics can be limited by lack of access to the source region or by difficulties in obtaining enough data to completely characterize the distribution function. Methods are therefore discussed for using simulation to infer characteristics of the beam distribution from the data that can be obtained.
\end{abstract}

\section{Introduction}

The precision normally associated with beam optics calculations in low-current accelerators has not always been observed in benchmarks against experiment when space charge is important.[1-3] Furthermore, the influence of space charge in the source region of a machine can be significant even when it is less important as the beam is accelerated. Even when space charge is not an important factor in the behavior of the bulk of an accelerator system, it can therefore still be important to include the influence of space charge in system design. To begin with, the characteristics of the source region determine the initial state of the beam, and characteristics of the initial state such as beam emittance and halo formation $[1,2,4]$ are often quite important to downstream behavior. 
Less apparent is that there are conditions where space-charge collective modes can be excited in the source region that persist for hundreds of focusing periods.

If one considers the transition from beams of low intensity where space charge can be neglected, as intensity is increased the consequences of space charge forces can often be correctly calculated by assuming a simple model. In this model the space charge contributes an average defocusing force which modifies the beam envelope evolution and modifies the various resonance frequencies. However, as intensity is increased further, a qualitative transition occurs in beam behavior and plasma-like collective modes[5-7] can become important to describing the beam evolution. Because the excitation of these modes depends on details of the beam distribution function in velocity space as well as configuration space, prediction of the evolution of these modes requires knowledge of the initial phase space characteristics that can be difficult to measure. In addition, variations in the "as-built" transport system characteristics associated with tolerances in construction and operating conditions can also have a significant effect on transport characteristics.

Many of the concepts developed to study nonneutral plasma [4,6] are applicable to the study of a space-charge-dominated beam. A parameter of importance to the beam dynamics is the ratio of beam radius, $r_{b}$, to the Debye length $\square_{\mathrm{D}}=\mathrm{v}_{\mathrm{th}} / \square_{\mathrm{p}}$, where, nonrelativistically, the beam kinetic energy is $\mathrm{mv}_{\mathrm{th}}{ }^{2}$ and the plasma frequency $\square_{\mathrm{p}}=$ $\left(4 \square \mathrm{ne}^{2} / \mathrm{m}\right), \mathrm{n}$ is the particle density, e the particle charge and $\mathrm{m}$ the particle mass. When this ratio $r_{b} / \square_{D}$ exceeds a factor of approximately two or three, the beam is able to support plasma-like oscillations, since such oscillations usually do not occur at wavelengths less than $\square$ D.

Description of the beam when it is space-charge-dominated has proved complex. For example, instabilities have been predicted theoretically using the idealized Kapchinskij-Vladimirskij [8] distribution [5,6,9,10], although the applicability of these instabilities to more realistic distributions has usually relied on simulations $[9,11,12]$. More recent work [7] has used a warm-fluid theory to demonstrate that the characteristics of many of the collective space-charge modes do not depend on details of the velocity distribution function. These modes, therefore, occur for more general distributions than the $\mathrm{K}-\mathrm{V}$ distribution originally used in their derivation. However, there still is little theoretical understanding of the mechanism by which these modes are damped, and simulations predict that some of these modes can persist for hundreds of focusing periods. It is therefore important to understand the excitation of these modes even for systems that are not space-charge-dominated for the most of their length.

\section{The University of Maryland Electron Ring (UMER) Source}

In view of complex phenomena found to occur in space-charge-dominated beams and the difficulty of understanding how to adequately model these phenomena, the UMER experiment is being constructed to study the fundamental physics of a spacecharge-dominated beam. Since much of this physics depends on details of the sixdimensional particle distribution function, it is important to adequately characterize the initial beam distribution. However, measurement of the detailed six-dimensional phasespace distribution is not usually practical. First-principle modeling of the beam source can therefore represent an important technique for inferring details of the beam distribution from the measurements that are possible. Such simulations are also an 
important technique for characterizing what level of description is required to adequately predict the downstream dynamics. Therefore, as the ring is being assembled and diagnostics tested, simulations are being performed to match existing data to extract as many details as possible about the beam characteristics.

Important questions about the sensitivity of the beam characteristics to small deviations from the nominal design can be addressed in some of these preliminary comparisons. These comparisons should therefore be important to understanding the operation of the injector, including any small deviations from the nominal design. As will be discussed below, some of the initial findings were not expected and therefore much of what is presented here is part of an ongoing program to refine our understanding of the UMER source operation.

The gun in UMER is a modified Pierce geometry with a $4 \mathrm{~mm}$ radius source, and is shown schematically in Fig. 1. The gun perveance can be varied by changing the voltage of the cathode-grid and by moving the anode plane. For the measurements discussed below, the anode-to-cathode distance was adjusted to approximately $25 \mathrm{~mm}$ so that the current obtained is the nominal $100 \mathrm{~mA}$ beam assumed in the ring design. One difference from standard pierce geometry is an anode-grid that transmits approximately $87 \%$ of the beam, and which was employed based on EGUN simulations that predicted a reduction in aberrations by ensuring that the anode plane is an equipotential surface. A more substantial deviation in operation from a standard Pierce diode results from a cathode-grid place $0.15 \mathrm{~mm}$ from the planar cathode surface. Since the separation between the $0.025 \mathrm{~mm}$ diameter wires in the square grid pattern is $0.15 \mathrm{~mm}$, i.e. the same as the distance to the cathode, the field pattern in the vicinity of the emitter surface is complex, especially when the self-consistent field of the space-charge-dominated beam is included. The cathode-grid is biased negatively relative to the cathode by a substantial voltage, usually of order $40 \mathrm{~V}$, to impede current flow until a positive pulse is applied to the cathode-grid to draw the pulse of current desired for ring injection. Note that because the cathode-grid pulser is at $10 \mathrm{kV}$ relative to ground it is difficult to precisely measure the grid pulse current and voltage when the beam is on.

A complete description of the extensive set of diagnostics being developed for measuring the dynamics of UMER is beyond the scope of this discussion. The primary diagnostic discussed here is a phosphor screen that can be moved along the injector transport line. A CCD camera is used to record the time-integrated image on that screen. The UMER gun structure also has a set of masks on a plate approximately $10 \mathrm{~mm}$ downstream of the anode plane that can be rotated to insert different masks allowing variation in the size or shape of the beam emerging from the gun, without breaking vacuum.

Five-Beamlet Emittance Measurements

A previous study of the beam evolution, in a long solenoid channel, where an electron beam passed through a mask with a quincunx pattern, (corresponding to the five spots on a die) revealed that the evolution of the patterns generated on a phosphor screen was sensitive to the beam emittance.[13] The original pattern was found to reemerge downstream from the original mask. Simulations of this experiment showed that the plane of the image, as well as the shapes of the intermediate patterns, depended on the 
emittance of the beamlets. This suggested that the five-beamlet mask could be used to estimate the beam emittance emerging from the UMER electron gun.

Figure 2 shows phosphor screen images obtained on the UMER injector $0.99 \mathrm{~m}$ and $1.04 \mathrm{~m}$ downstream from a five-beamlet mask $10 \mathrm{~mm}$ downstream of the anode plane. These patterns are typical of what is observed as the masked beam propagates. The planes shown were chosen because they are at the limit of the straight distance that was available in the injector transport line, and since the dependence of pattern details on emittance tends to exhibit maximum sensitivity when the beam has propagated to near the plane of where the image is formed. Also shown on the same figure are simulations performed using the WARP PIC code [14] in single slice mode. In this mode the code solves the two-dimensional transverse evolution of the beam using $\mathrm{s}$, the distance along the transport line, as the independent variable. In these simulations the initial beam before transiting the mask is assumed uniform across the beamlets and the velocity distribution is assumed to be Gaussian and with constant temperature across the beam. The emittances labeled on the simulation curves shown in Fig. 2 are calculated by multiplying the thermal velocity assumed for the beamlets by the total area of the beam at the anode plane in the absence of the mask. From a comparison between the simulated and measured pattern it is inferred that the best match occurs when the initial normalized emittance of the total beam is between $16 \square \mathrm{m}$ and $18 \square \mathrm{m}$. This is compared to approximately $3.6 \square \mathrm{m}$, which is the intrinsic emittance of the source calculated by multiplying the source radius by the thermal velocity corresponding to a cathode temperature of $0.1 \mathrm{eV}$. There are several mechanisms that can cause the source emittance to exceed the intrinsic value. However, because masking the beam at the anode plane tends to reduce the emittance growth that results from aberrations in the gun structure, the likely cause of the bulk of the emittance growth is thought to result from the perturbation to the beam distribution by the cathode-grid.

Upstream Beam Measurements

Figure 3 is a set of pictures taken with the phosphor screen positioned approximately $60 \mathrm{~mm}$ from the anode plate. The leftmost column contains phosphor screen images of the full beam taken at different settings of the bias voltages. The center column is a set of plots of the light output, which is an approximately linear function of the local current density, along a diameter of the plots in the first column. The right column is a set of phosphor screen pictures at the same location but with a "pepper-pot" mask. Such a mask consists of holes that are sufficiently small that the emerging beam is emittance dominated. The particles in the beam then follow straight-line orbits until they intercept the phosphor screen, so that the light intensity on the screen reflects the transverse velocity distribution at the location of each of the small holes in the mask.

It should be recalled that a DC bias voltage is applied between the cathode-grid and the cathode to retard any current until the voltage pulse is applied. While it would be possible to adjust the amplitude of the applied pulse, it is simpler to vary the cathode-grid to cathode voltage during the pulse by adjusting the bias voltage. As the bias voltage is increased from the minimum value needed to prevent any cathode current flow, the absolute voltage of the rectangular voltage pulse applied to turn on the current is reduced. At the lowest voltage, therefore, the maximum pulse voltage is applied to the cathodegrid and it is expected that the cathode-grid perturbation of the beam velocity distribution 
would be maximized. As the voltage is increased, the cathode-grid to cathode potential difference comes closer to the value it would float at if it were not energized, and therefore the cathode-grid perturbation on the particle distribution is expected to be somewhat reduced. One of the surprising features of the pepper pot measurement is the observed hollowing in the velocity distribution most evident at the lowest bias voltage of $45 \mathrm{~V}$ and still evident at $50 \mathrm{~V}$. This is an apparent indication that the cathode-grid is strongly perturbing the initial particle distribution by imparting a substantial amount of transverse directed energy to the majority of the beam particles. As the bias voltage is increased so that the cathode-grid to cathode potential is reduced, this energy is seen to decrease so that at the highest bias voltage, the velocity distribution begins to more closely resemble the transverse Gaussian distribution expected from a thermionic cathode surface. Another feature of note is that at the intermediate bias voltages, an intermediate velocity distribution is observed which does not exhibit as much hollowing but shows a squaring of the velocity distribution that presumably reflects the square grid pattern.

Simulation of the entire gun is not straightforward. The geometry becomes threedimensional because the axisymmetry is broken by the rectangular grid pattern, and there is added complexity introduced by the disparity in scales between the $0.15 \mathrm{~mm}$ dimension characteristic of the grid pattern and the $25 \mathrm{~mm}$ distance from the cathode-grid to the anode-grid. The simulations discussed here have therefore first concentrated on consideration of a model that does not attempt to directly model the complex behavior in the cathode-grid region, but instead starts the simulation by injecting a specified beam distribution injected at the cathode-grid plane. This distribution is assumed to be transversely uniform, so that no attempt has yet been made to include the direct interception of the beam current by the cathode-grid wires occupying $31 \%$ of the grid area. This direct interception is spatially correlated with the transverse acceleration of the beam particles and therefore affects the phase of any short wavelength space-charge oscillations launched by the cathode-grid. Inability to get direct agreement between the simulated and measured distributions at the plane of the pepper-pot mask is believed to be a consequence of this approximation in the model. Emphasis was instead placed on the spatial characteristics of the full-beam distribution and an exhibition, using the simulations, of how this distribution could be a sensitive function of details of the assumed initial velocity distribution. This is contrary to the normal assumption that, because the kinetic energy in a space-charge dominated beam is much smaller than the space-charge potential energy in a nonuniform beam $[15,16]$, the transverse cross section should not be sensitive to details of the velocity distribution.

Figure 4 is a plot of the transverse current distribution of the simulated beam integrated to the plane of the phosphor screen measurements shown in Fig. 3. The distribution injected at the plane of the cathode-grid is spatially uniform and with a uniform Gaussian velocity distribution with a thermal spread corresponding to the $0.1 \mathrm{eV}$ cathode temperature. This beam is therefore injected at what is known as the inherent, or intrinsic, emittance. The transverse density variation of the beam integrated to the measurement plane was found to be insensitive to the injection energy, which was nominally fixed at $20 \mathrm{eV}$. The relative unimportance of any transverse variation which would occur in a self-consistent calculation was demonstrated by running a simulation which calculated the gun behavior by assuming space-charge-dominated emission at the 
injection plane. When this was compared to a transversely uniform injected beam with the same total current very little difference was observed.

The simulation shown in Fig. 4 was performed using the WARP code, referenced above. The r-z module was used to calculate the full time evolution in the region between the cathode-grid and the anode-grid. After reaching steady state, the beam particles were collected at the anode-grid, the current was reduced to reflect the approximately $87 \%$ anode-grid transparency. The beam was then propagated using the two-dimensional single-slice model for the remaining $60 \mathrm{~mm}$ to the measurement plane. It is worth noting that the rms emittance in the simulation calculated at the plane of the anode-grid is approximately $6 \square \mathrm{m}$ compared with the injected intrinsic emittance of approximate $3.6 \square \mathrm{m}$. This presumably reflects the aberrations in the gun structure.

The profile in Fig. 4 is quite different from what is observed on the phosphor screen. Since the cathode-grid is known to perturb the transverse velocity distribution, simulations were performed to examine the consequences to the profile from an increase in the assumed value of the initial thermal spread. Figure 5 shows the change in profile at the downstream measurement plane when the initial thermal velocity is five times the intrinsic value. The rms emittance measured from the simulation was approximately 25 $\square \mathrm{m}$, which is more than the $16 \square \mathrm{m}$ to $18 \square \mathrm{m}$ inferred from the five-beamlet experiment. The central uniform region in this profile does not match the measured profiles for the $100 \mathrm{~mA}$ beams, but does resemble the profile measured at the $55 \mathrm{~V}$ bias. At this voltage the velocity distribution measured by the pepper pot is a beginning to look Gaussian. Since the beam at this bias voltage only has a current of $81 \mathrm{~mA}$ compared with the approximately $100 \mathrm{~mA}$ for the other bias voltages, it is not clear how to compare the simulations to the data. Because the beam evolution is not a simple function of the current, it will be necessary to rerun a series of simulations at the lower current in order to explore the sensitivities of the output distribution to the assumed input distribution function at the lower current.

By increasing the injected thermal velocity by another factor of two, to ten times the intrinsic thermal velocity, the current profile shown in Fig. 6 is obtained which somewhat resembles the measured distribution. However the calculated rms emittance of $49 \square \mathrm{m}$ at the anode plane is now substantially higher than the emittance inferred from the five-beamlet comparison.

Because the potential energy associated with space charge is normally much greater than the kinetic energy in a space charge dominated beam, it is normally assumed that the transverse density distribution will not be sensitive to the form of the velocity distribution. For example the matched rms beam radius in a transport line will usually remain constant over a wide range of emittance if the beam is sufficiently space-charge dominated. However, motivated by the measurements which indicated that the velocity distribution was far from the normally assumed Gaussian shape, it was decided to run simulations with an initially "double-humped" distribution in the $\mathrm{x}$ and $\mathrm{y}$ directions. That is the distributions for $n\left(v_{x}\right)$ and $n\left(v_{y}\right)$ were constructed by displacing four subspecies by $\pm \square v_{\text {th }}$ in $v_{x}$ and $v_{y}$, where both the constant $\square$ and $v_{\text {th }}$, the width of the assumed Gaussian distribution, were varied to investigate the consequence to the shape of the output distribution measured at the plane of the phosphor screen. It should be noted that even though the assumed form of the distribution, which would appear as a fuzzy square on a pepper pot measurement, breaks the axisymmetry, this breaking occurs only in velocity 
space. Full three-dimensional simulations, which were run to check any apparent inconsistency between the symmetries, yielded results almost identical to the r-z runs.

Figure 7 is a plot of the radial variation in current density for a distribution with $\square$ $=4.0$ and $v_{\text {th }}$ twice the intrinsic value. Note that the shape more closely resembles the measured shape shown in Fig. $3 \mathrm{f}$ than the calculated curves shown in Fig. 4-6. This is despite the fact the rms emittance for this distribution measured at the anode plane is only $12.5 \square \mathrm{m}$, which is approximately half of the emittance in Fig. 5. It should be noted that the shape of the profile is a sensitive function of both $\square$ and $v_{\text {th. }}$. This would seem to be an indication that the particular shape observed is composed of radial sections of the beam that are not traveling with linearized radial velocities during traversal of the gun. This type of deviation from laminar is not an unusual feature of finite-temperature gun optics.

An interesting feature of the simulations represented by the plot in Fig. 7 is the small dip near the center of the beam that persistently appears over a relatively wide range of parameters, but is only slightly evident in the plot of density along the cut across a beam diameter for the $45 \mathrm{~V}$ data in Fig. 3. Careful examination of the full twodimensional phosphor image, however, does reveal a dip in density near the center of the beam that is not clearly seen in the plot of experimental density distribution because it is slightly off-center. Note that this dip is seen only when a high quality print of the data is generated. It is also possible that the same small misalignment that causes the hole to be off-centered also diminishes the magnitude of the dip relative to the simulations that assume axisymmetry.

\section{Conclusions}

Simulations have been presented which attempt to infer the consequences of the distortion of the injected distribution function by the cathode-grid employed in the UMER gun to control the beam current. While the simulations lack some of the relevant physics, particularly the consequences of the configuration space modulation of the beam by the cathode-grid and the correlations of this modulation to the velocity space distortions, many features of the beam characteristics can be recovered by a combination of the simulations and the indirect measurement. Particularly noteworthy is the sensitivity of the radial shape of the beam density variation at the output from the gun. This sensitivity is not normally observed in a space-charge-dominated transport line.

Further simulations are planned to explore the beam behavior in the cathode-grid to cathode region by doing high-resolution numerical calculations that attempt to examine the behavior of the beam from first principles. Taken together with further measurements in the vicinity of the gun output, it should be possible to infer details of the evolution of the full six-dimensional beam distribution in the gun region that are not normally measured. Since there are many devices whose operation depends strongly on the characteristics of the beam emerging from the source, a more detailed understanding of the evolution of the six-dimensional distribution function should promote designs tht result in improved beam characteristics. 
LBNL-57119

HIFAN 1404 


\section{References}

[1]. T. P. Wangler, C. K. Allen, K. C. D. Chan, P. L. Colestock, K. R. Krandal, R. W. Garnett, J. D. Kilpatrick, W. Lysenko, J. Qiang, J. D. Schneider, M. E. Sculze, R. L. Sheffield, and H. V. Smith, "Beam Halo in Mismatched Proton Beams," proc. of this conf

[2]. J. Qiang, P. L. Colestock, D. Gilpatrick, H. V. Smith, T. P. Wangler, M. E. Schulze, "Macroparticle simulation studies of a proton beam halo experiment," PRST 5, 124201 (2002).

[3]. S. M. Lund, J. J. Barnard, G. D. Craig, A. Friedman, D. P. Grote, H. S. Hopkins, T. C. Sangster, W. M. Sharp, S. Eylon, T. J. Fessendon, E. Henestroza, S. Yu, I. Haber, "Numerical Simulation of Intense-Beam Experiments at LLNL and LBNL," Nucl. Instr. and Meth, in Phys Res. A, 415,345 (1998).

[4]. Martin Reiser, Theory and Design of Charged Particle Beams, (John Wiley and Sons, Inc., New York, 1994).

[5] R. L. Gluckstern, "Oscillation Modes in Two Dimensional Beams," Proc. of the Linac Conference, Fermilab, 1970, p811.

[6]. Ronald C. Davidson, Physics of Nonneutral Plasmas, (Addison-Wesley, Redwood City, CA, 1990).

[7]. Steven M. Lund, Ronald C. Davidson, "Warm Fluid Description of Intense Beam Equilibrium and Electrostatic Stability Properties, Phys. Plasmas, 5, 3028 (Aug. 1998).

[8]. I. M. Kapchinskij and V. V. Vladimirskij, "Limitations of Proton Beam Current in a Strong Focusing Linear Accelerator with Beam Space Charge," Proc. Int. Conf. on High Energy Accelerators and Instrumentation, ed. by L. Kowarski, p. 274 (CERN, Geneva, 1959).

[9]. I. Hofmann, L. J. Laslett, L. Smith and I. Haber, "Stability of the KapchinskijVladimirskij (K-V) Distribution in Long Periodic Transport Systems", Particle Accelerators 13, 145 (1983).

[10]. T. F. Wang and L. Smith, “, "Transverse-Longitudinal Coupling in Intense Beams," Particle Accelerators, 12, 247, (1982).

[11]. I. Haber, "Space Charge Limited Transport and Bunching of Non K-V Beams", IEEE Tran. Nucl. Sci. NS-26, 3090. (1985).

[12]. I. Haber, A. Friedman, D. P. Grote, S. M. Lund, and R. A Kishek, "Recent Progress in the Simulation of Heavy Ion Beams," Phys. Plasmas, Vol. 6, (May, 1999) 2254. 
[13] I. Haber, D. Kehne, M. Reiser, and H. Rudd, "Experimental, Theoretical and Numerical Investigation of the Homogenization of Density Nonuniformities in the Periodic Transport of a Space-Charge Dominated Beam," Phys. Rev. A15, 44, 5194 (Oct. 15, 1991).

[14] D. P. Grote, A. Friedman, I. Haber, W. Fawley, J. L. Vay, "New Developments in WARP: Progress Toward End-to-End Simulation," Nucl. Instr. and Meth, in Phys Res. A, 415, 428 (1998).

[15] M. Reiser, J. Struckmeier, J. Klabunde and M. Reiser, "On the Stability and Emittance Growth of Different Phase-Space Distributions in a Long Magnetic Quadrupole Channel", Particle Accelerators 15, 1344 (1984).

[16] T. P. Wangler, K. R. Crandall, R. S. Mills, and M. Reiser,"Relation Between Field Energy and RMS Emittance in Intense Particle Beams," IEEE Trans. Nuc. Sci. NS32, 2196 (1985). 
Figure Captions

Fig. 1. Schematic rendering of the UMER gun, which is a Pierce geometry source modified by the inclusion of a cathode-grid, G, $0.15 \mathrm{~mm}$ from the cathode, $\mathrm{K}$, and an anode-grid at the anode plane, A. The cathode-grid consists of $0.0025 \mathrm{~mm}$ wires in a square pattern with $0.015 \mathrm{~mm}$ spacing between the wires and is used to control the gun current. The anode-grid is used to maintain an equipotential surface at the anode plane.

Fig 2. Phosphor screen data taken 0.99 and $1.04 \mathrm{~m}$ from the anode plane when the beam is passed through a mask near the anode plane that creates a pattern of beam current of five beamlets. The initial pattern resembles the five dots on a die. Also shown are a series of simulations that vary the initial temperature to determine what initial temperature range will match the measured pattern. The labels on the simulation curves correspond to the normalized emittance for the full beam with the temperature of the beamlets.

Fig. 3. Phosphor screen data, at a plane $60 \mathrm{~mm}$ from the anode, for different values of the bias voltage on the cathode-grid. The left column is a picture of the full beam. The center column is of an intensity scan across a beam diameter. The right pattern is the phosphor screen pattern generated by a pepper pot mask near the anode plane and therefore measures the velocity distribution at each of the holes in the pepper pot mask. Note the mm scale of the distances in the density scans, that are not labeled on the plot.

Fig. 4. Plot of the beam density vs. radius of a simulated beam at the same $60 \mathrm{~mm}$ plane as the data in Fig. 2. The initial distribution injected at the cathode-grid plane is transversely uniform in density and Gaussian in velocity distribution with a spread corresponding to the $0.1 \mathrm{eV}$ intrinsic cathode temperature.

Fig. 5. Beam density vs. radius for a simulation similar to the plot in Fig. 4 but the thermal spread of the initial Gaussian distribution has been increased by a factor of five.

Fig. 6. Beam density vs. radius for a simulation similar to the plot in Fig. 3 but the thermal spread of the initial Gaussian distribution has been increased by another factor of two so that it is ten times the value in Fig. 4.

Fig. 7. Beam density vs. radius for a simulation with an initial distribution which is "double-humped" in the $\mathrm{x}$ and $\mathrm{y}$ directions. The initial beam is constructed as the sum of four subspecies that are uniform in configuration space. However each of the subspecies has a thermal spread twice the intrinsic value, but each is offset by $\pm 4.0 \square \mathrm{v}_{\mathrm{th}}$, i.e. four times this thermal spread, in both $\mathrm{v}_{\mathrm{x}}$ or $\mathrm{v}_{\mathrm{y}}$. 
LBNL-57119

HIFAN 1404 


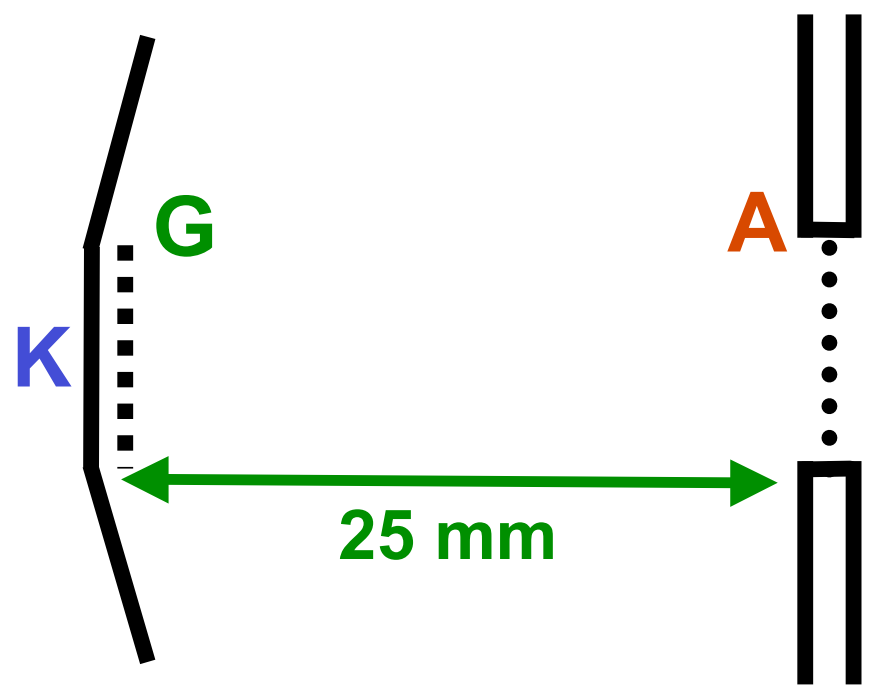




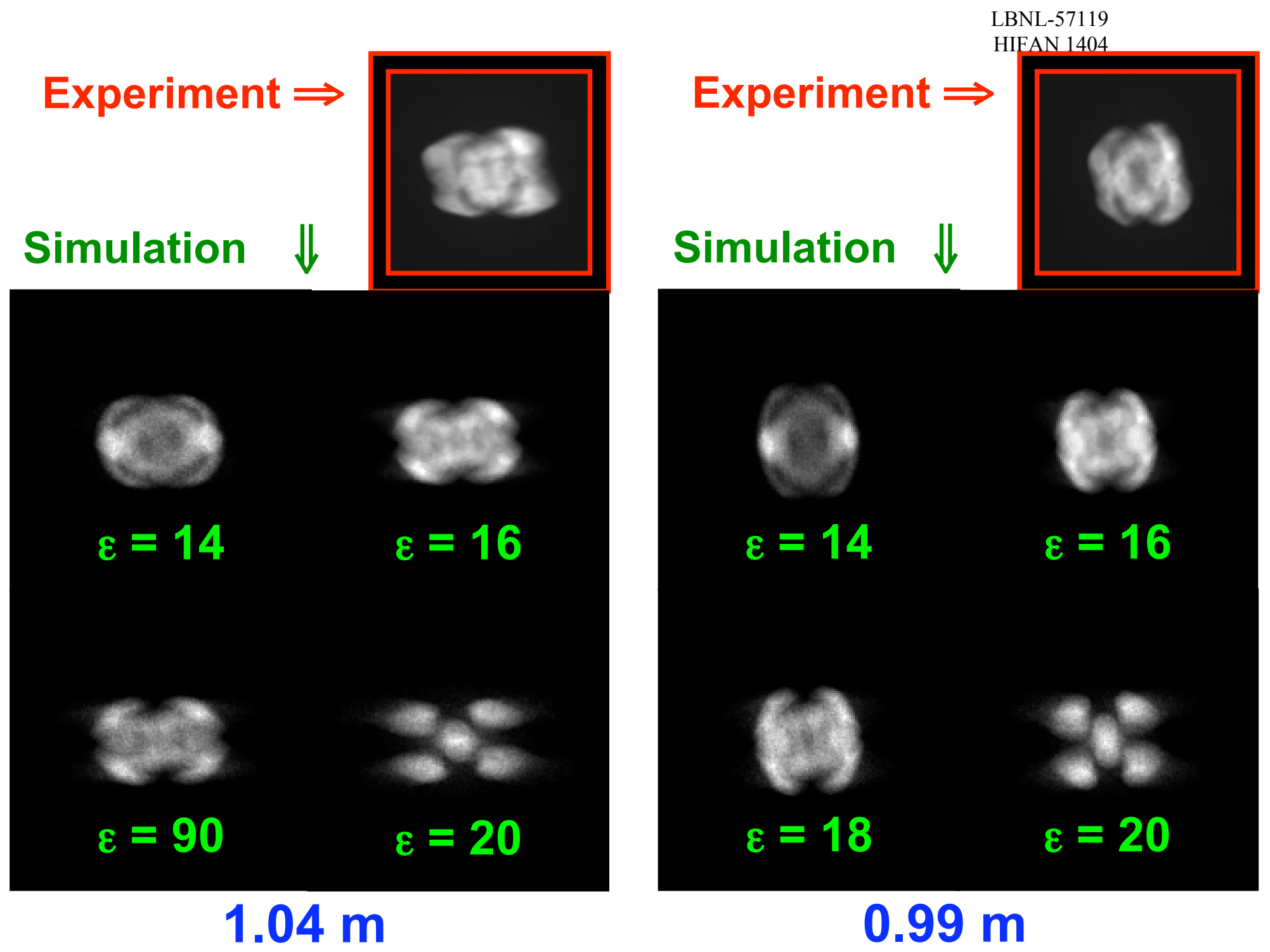


$45 \mathrm{~V}$
$(101 \mathrm{~mA})$

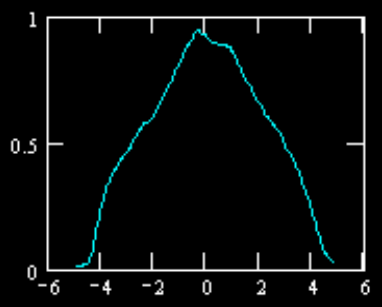

$50 \mathrm{~V}$

(100.8 mA)

$52 \mathrm{~V}$

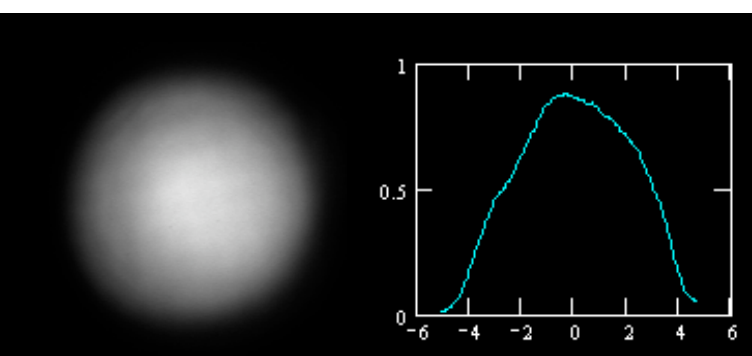

$55 \mathrm{~V}$

(81 mA)
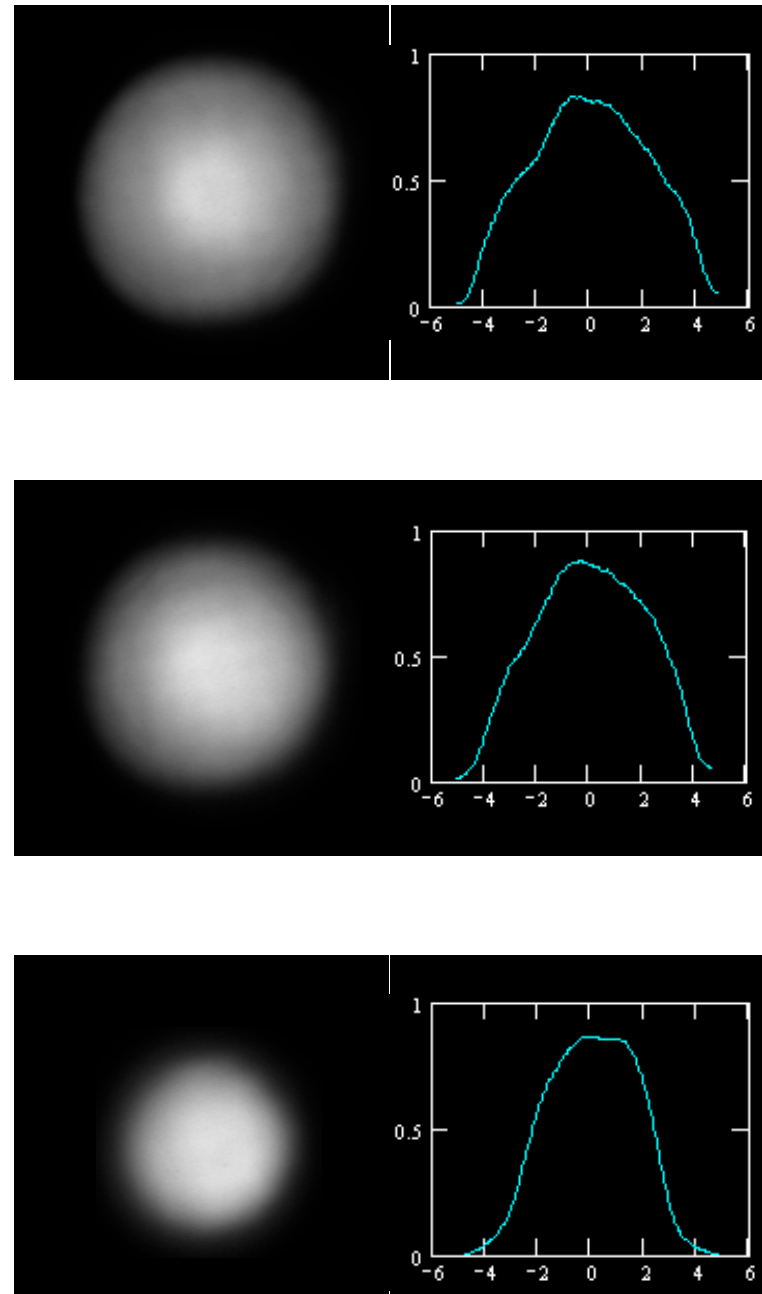


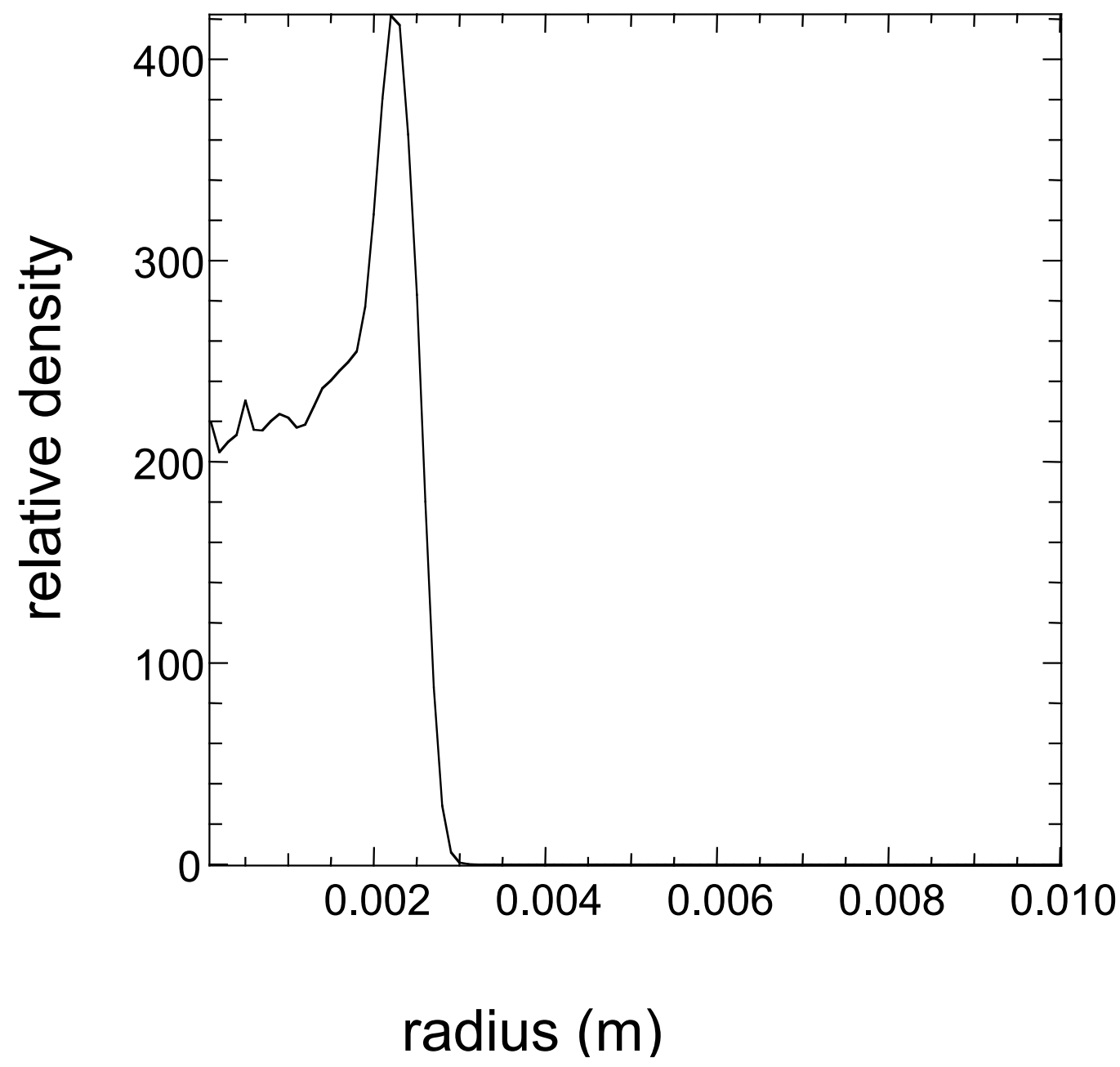




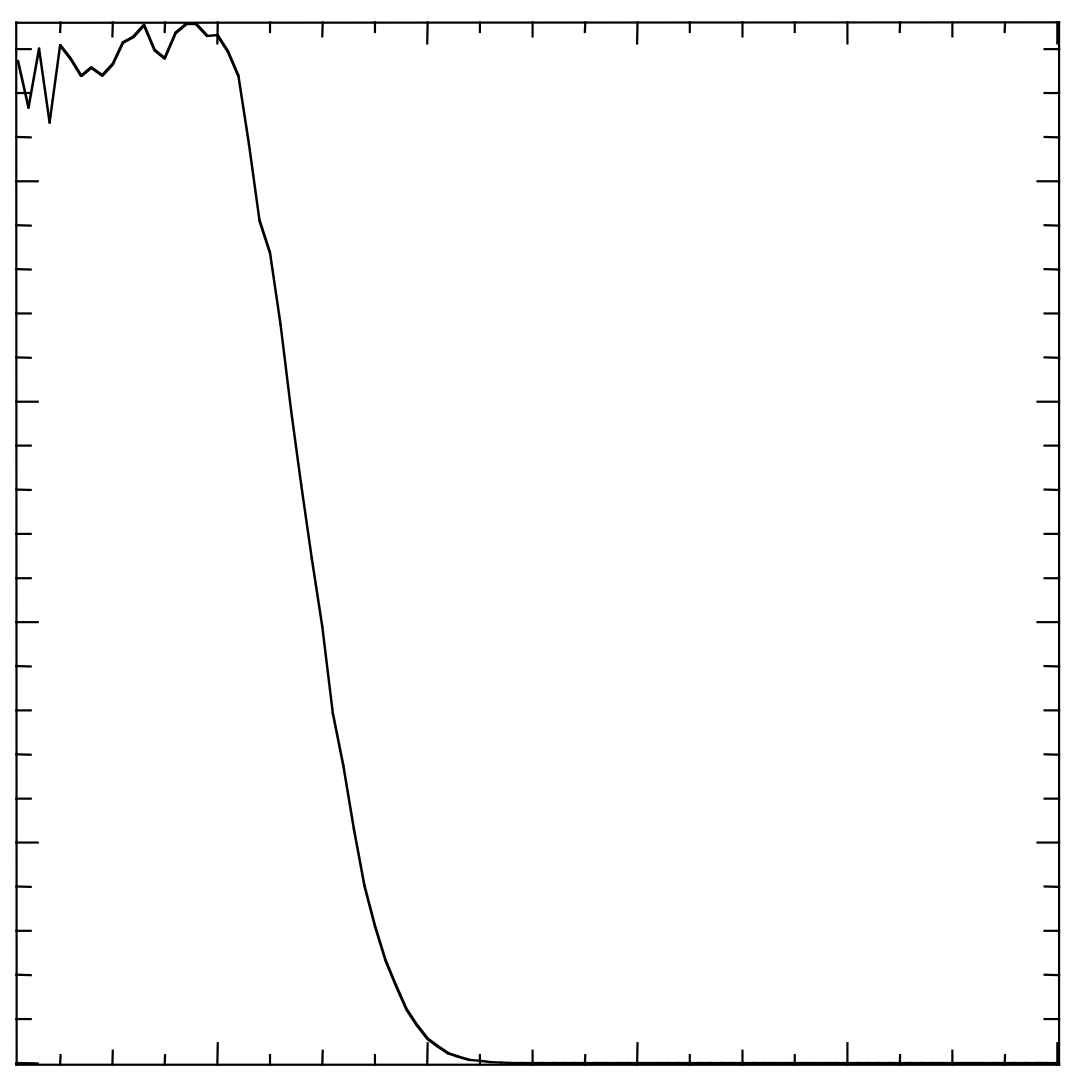




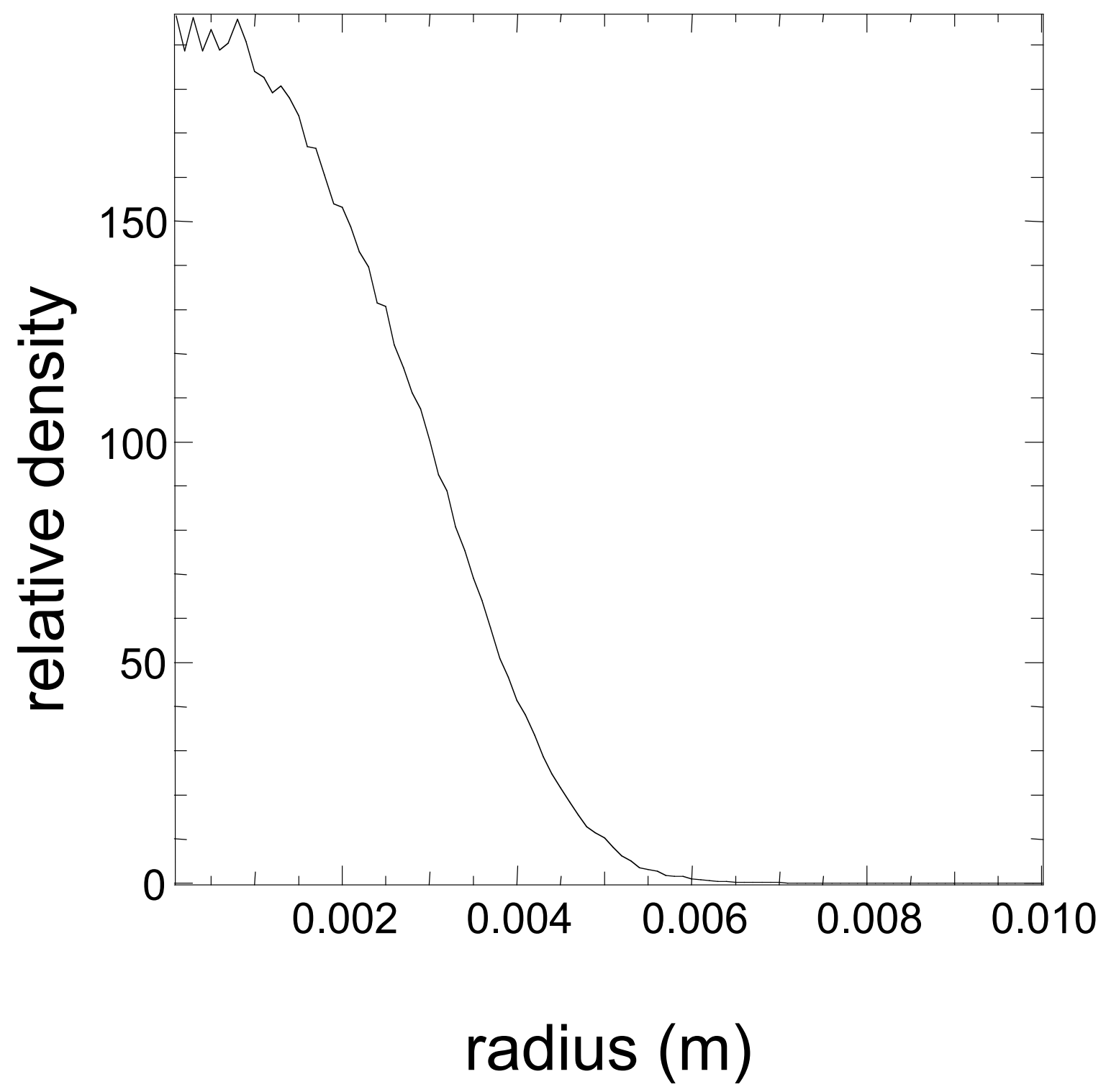




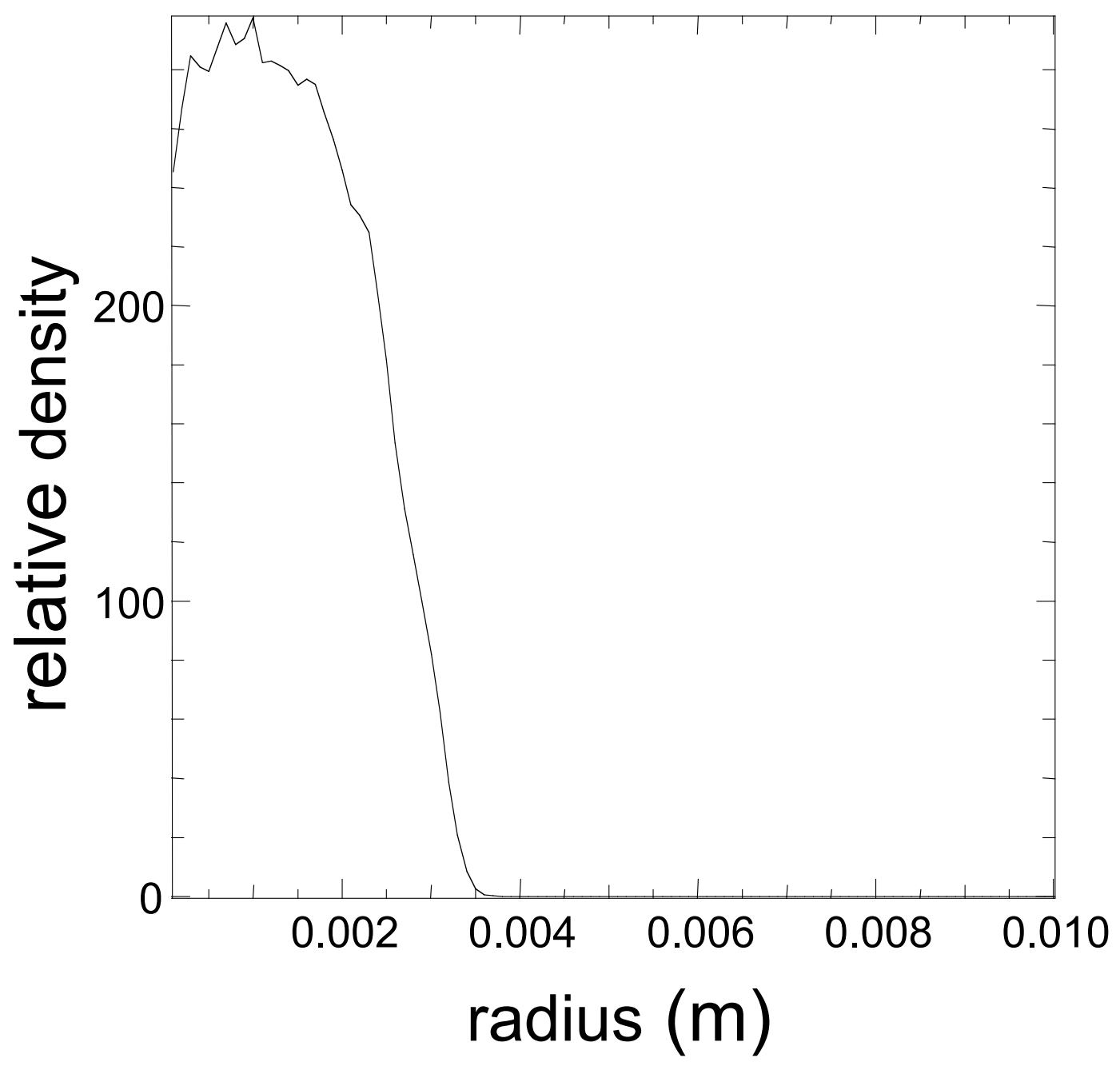

